\section{Nutritional transition: a public health challenge in developing countries}

\section{S K Kapoor, K Anand}

\section{The double burden of undernutrition and overnutriton in developing countries is a public health challenge}

D eveloping countries are undergoing various types of transitions. The epidemiological transition makes them face a double burden of communicable and non-communicable diseases. Similarly, demographic and socioeconomic transitions are also occurring in these countries. Earlier developing countries had a high prevalence of undernutrition, but this era of transition has also brought a double burden of undernutrition and overnutrition in these countries. Shukla et al in their descriptive study of body mass index (BMI) in western India in this issue of the journal have demonstrated this burden.

BMI, also known as Quetelet's index, relates height to weight and is measured by the formula-weight $(\mathrm{kg}) /$ height $(\mathrm{m})^{2}$. It has been recommended to use this index as it is difficult to measure fat mass under field conditions. For different grades of overweight among adults, an expert committee of the World Health Oranisation has recommended three cut off points of 25, 30, and 40 .

While interpreting these cut off points, the following points should be kept in mind ${ }^{2}$ :

- The recommended cut offs are appropriate for identifying the extent of overweight in people, but do not imply targets for intervention

- Weight gain in adult life may be associated with increased morbidity and mortality independently of the original degree of overweight.

- These cut off points should not be interpreted in isolation but must be interpreted with other determinants of morbidity and mortality (disease, smoking, blood pressure, etc)

Why has the focus been on screening overweight people? Because:

- Increased weight gain increases morbidity and mortality

- Overweight is associated with increased morbidity and mortality

Dudeja and coauthors in their study of 123 healthy volunteers commented that conventional cut off values of the BMI $\left(25 \mathrm{~kg} / \mathrm{m}^{2}\right)$ in identifying subjects who were overweight has a low sensitivity and negative predictive values as compared with cut off values based on percentage of body fat (male $>25$, female >30). ${ }^{3}$ They have based their opinion on findings that Asian Indians are at a higher risk for the development of atheroscelerosis and related complications possibly initiated by higher body fat. The cut off points as advocated by them for Asian Indians were $23 \mathrm{~kg} / \mathrm{m}^{2}$ for overweight and $25 \mathrm{~kg} / \mathrm{m}^{2}$ for obesity.

Like overweight, marked thinness (underweight) is also responsible for higher morbidity and mortality. ${ }^{4}$ In an Indian study men who had a BMI of less than 18.5 had a progressive increase in mortality rate. Those with BMI less than 16 had threefold higher mortality rate. ${ }^{4}$

As reported by Shukla et al in this issue both thinness and overweight are present in western India. A similar situation has been reported from other parts of India. In our study in suburban area of north India (unpublished data) the prevalence of thinness was $16.4 \%$ whereas overweight was above $28 \%$. The existence of double burden of undernutrition and overnutrition has been witnessed in Brazil in a very low income population. In adults obesity was present in $25 \%$. Stunting was also present in $22 \%$ of adults; of these $30 \%$ were overweight obese and $16.3 \%$ were underweight. Another study reported coexistence of overweight and underweight in the same households of three countries-China, Brazil, and Russia. ${ }^{6}$ The reported percentage of households where persons with undernutrition and overnutrition existed simultaneously, the proportion was found to be highest in Brazil, which is undergoing rapid transition.

The epidemiological transition leading to higher prevalence of noncommunicable diseases is associated with overweight and obesity. The various factors, which may be responsible for higher prevalence of obesity even in developing countries, are urbanisation and life style factors like lower energy expenditure labour, mechanisation, and automation. This also includes watching television and other sedentary activities. Changes in dietary habits like fast food culture, increase in energy density of diet (more \% of fat), and consumption of more meals outside the home.

The existence of a double burden poses a challenge for intervention. The overweight and obesity makes people prone to non-communicable and degenerative diseases whereas undernutrition may make them prone to communicable diseases and reduce productivity. As these may be coexisting, not only at country level but also even at household level, no uniform intervention strategy can be advocated. Obviously this calls for a closer sociological look at factors affecting nutrition within households.

Ever since Geoffrey Rose's seminal paper on "Sick individuals and sick populations $^{\prime \prime}$ the appropriate public health approach for any chronic disease prevention is health promotion. However applying this approach to nutrition results in a paradox in a country that is in nutritional transition. The approach of health promotion has been on shifting the entire distribution of factor under consideration to right or left as may be a desirable end point. This approach is valid only if one sided extreme is of major importance, for example, hypertension compared with hypotension. In the field of nutrition, data available indicate that both undernutrition and overweight are undesirable entities. Therefore, by shifting the curve to one side, while we may achieve reduction of one undesirable extreme, we end up increasing the other one. Perhaps it is the time to consider exceptions to Rose's postulates. In a recent revisit to Rose's theory, soundness of basic approach was taken for granted by commentators. ${ }^{10}$ However, it is only recently that some data are being presented to prove the theory of Rose.

Discussing Rose's theory, however does not help us in tackling the basic issue of public health approach towards tackling undernutrition and overweight simultaneously. It is recommended that the ideal mean BMI for a population in transition might be between $21-23 \mathrm{~kg} /$ $\mathrm{m}^{2}$, a level that is currently existing. Therefore, we have to tackle both the extremes of curve by targeted interventions rather than by a whole population based approach. The targeting might be at multiple levels from individuals to specific communities. Targeting could also mean focus on undernutrition in children and overnutrition in adults. This is in keeping with the theory of undernutrition in childhood being responsible for adult non-communicable diseases. Tackling childhood malnutrition could prevent stunting, thereby increasing adult heights as well. This would automatically shift the adult BMI curve to left, thereby reducing overweight prevalence. While childhood obesity is not yet a major problem in developing countries, this also would need to be kept in mind. 
The importance of the paper by Shukla et al is in opening this Pandora's box that raises more questions and provides few answers. We may also need to have a relook at the validity of cut off points for the BMI used for defining various states of nutrition in developing countries undergoing nutritional transition.

$J$ Epidemiol Community Health 2002;56:804-805

\section{Authors' affiliations}

S K Kapoor, K Anand, Comprehensive Rural Health Services Project, Ballabgarh, All India

Institute of Medical Sciences, District

Faridabad-121004, Haryana, India
Correspondence to: Professor S K Kapoor: crhspaiims@sancharnet.in

\section{REFERENCES}

Shukla HC, Gupta PC, Mehta HC, et al. Descriptive epidemiology of body mass index of an urban adult population in western India. $J$ Epidemiol Community Health 2002;56: 876-80.

2 World Health Organisation. Physical status: the use and interpretation of anthropometry. Technical Report Series no 854. Geneva: WHO, 1995:312-70.

3 Dudeja V, Mishra A, Pandey RM, et al. BM does not accurately predict overweight in Asian Indians in northern India. $\mathrm{Br} J$ Nutr 2001;86:105-12.

4 Naidu AN, Rao NP. Body mass index: a measure of nutritional situation in Indian Population. Eur J Clin Nutr 1994;48 (suppl 3) S131-40.
5 Yap MD, Yian TB, Kai CS, et al.

Manifestations of cardiovascular risk factors at low level of body mass index and waist hip ratio in Singapore Chinese. Asia Pacific Journal of Clinical Nutrition 1999:8: 172-83.

6 de Nevezer TM, Florencio T, da Silva Ferregia $\mathrm{H}$, et al. Obesity and undernutrition in a very low income population in the city of Maceid, northern Brazil. Br J Nutr 2001;86 277-88.

7 Doak CM, Adair LS, Moneteiro C, et al. Overweight and underweight coexist within household in Brazil, China and Russia. J Nutr 2000;130:2965-71.

8 Caballero B. Obesity in developing countries: biological and ecological factors. J Nutr 2001;131:866-70S.

9 Rose G. Sick individuals and sick populations. Int J Epidemiol 1985;14:32-8

10 Davey Smith G, Ebrahim S. Reiterations: sick individuals and sick populations. Int Epidemiol 2001;3:427-42.

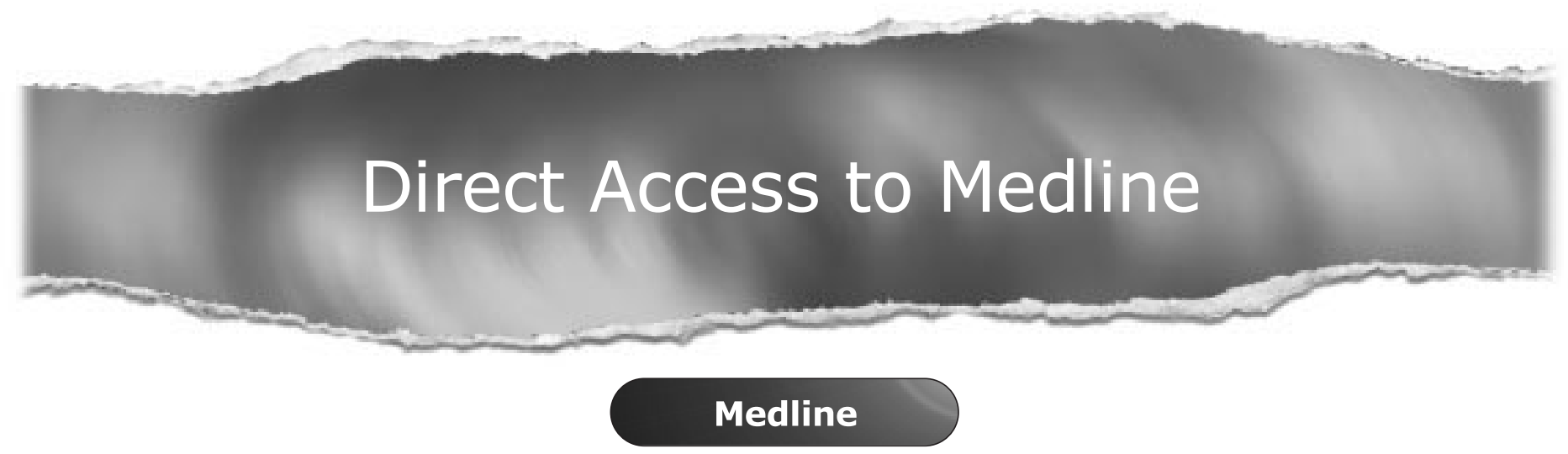

Link to Medline from the homepage and get straight into the National Library of Medicine's premier bibliographic database. Medline allows you to search across 9 million records of bibliographic citations and author abstracts from approximately 3,900 current biomedical journals.

www.jech.com 